\title{
Carvalho K manuscript
}

Katherine Carvalho ${ }^{1}$, Priscila Elias Ferreira Stricker ${ }^{1}$, Daiany de Souza ${ }^{1,2}$, Ana Carolina Irioda $^{1}$, Bassam Felipe Mogharbel ${ }^{1}$, Celia Regina Cavichiolo Franco ${ }^{3}$, José Roberto de Souza Almeida Leite, ${ }^{2}$, Alyne Rodrigues de Araújo ${ }^{4}$, Rondinelli Donizetti Herculano ${ }^{5}$, and Carlos Frederico de Oliveira Graeff ${ }^{6}$

${ }^{1}$ Pelé Pequeno Príncipe Institute, Child and Adolescent Health Research and Pequeno Príncipe Faculty ${ }^{2}$ Brasília University, Brasilia, Federal District - Brazil.

${ }^{3}$ Federal University of Paraná, Curitiba - Paraná - Brazil.

${ }^{4}$ Federal University of Piauí, Parnaíba - Piauí - Brazil.

${ }^{5}$ São Paulo State University (UNESP), Araraquara - São Paulo - Brazil.

${ }^{6}$ São Paulo State University (UNESP), Bauru - São Paulo - Brazil.

July 6, 2020

\section{Hosted file}

Carvalho K manuscript.pdf available at https://authorea.com/users/339930/articles/467074carvalho-k-manuscript 